OPEN

SUBJECT AREAS:

SELF-ASSEMBLY

CHEMISTRY

Received

17 September 2014

Accepted

26 November 2014

Published

22 December 2014

Correspondence and requests for materials should be addressed to

Y.Y. (yunyan@pku. edu.cn) or J.H.

(jbhuang@pku.edu.cn)

\section{Self-Assembly of Channel Type $\beta$-CD Dimers Induced by Dodecane}

\author{
Chengcheng Zhou ${ }^{1,2}$, Xinhao Cheng' , Qiang Zhao', Yun Yan' , Jide Wang ${ }^{2} \&$ Jianbin Huang'
}

'Beijing National Laboratory for Molecular Sciences (BNLMS), State Key Laboratory for Structural Chemistry of Unstable and Stable Species, College of Chemistry and Molecular Engineering, Peking University, Beijing 100871, China, ${ }^{2}$ College of Chemistry and Chemical Engineering, Xinjiang University, Urumqi, 830046, China.

Cyclodextrins (CDs) can hardly self-assemble into well-defined structures. Here we report if they preassemble into channel type dimers assisted by dodecane, well-defined vesicles and bricks can be formed. Unlike the traditional self-assembly of amphiphilic molecules driven by hydrophobic effect, the self-assembly of the channel type dodecane@2 $\beta$-CD supramolecular building block is predoninantly driven by hydrogen-bonds. More water molecules are found in the lyophilized vesicles than in the bricks, suggesting water molecules play an important role in the self-assembly of the channel-type dimers of $\beta$-CD. The amount of structural water in the self-assembly is closely related to the curvature of the final self-assembled structures. Our work reveals that the channel-type dimer of $\beta-C D$ may represent a new sort of building block for advanced structures.

M olecular self-assembly has been verified a powerful protocol to fabricate a rich variety of nanostructures $^{1-3}$. In most cases, hydrophobic effect occurs in self-assembling systems, such as the selfassembly of surfactants, membrane lipids, and other amphiphiles ${ }^{3,4}$. Recently, however, the nonamphiphilic self-assemblies, especially vesicles, are attracting considerable attention owing to their contribution to the fundamental science, especially to the understanding of the driving forces for molecular self-assembly ${ }^{5-7}$. It has been found that the nonamphiphilic vesicles can be driven by electrostatic interactions, dipole interactions, and van der Waals interactions, etc. A typical example is the self-assembly of polyoxometalate macroions ${ }^{5}$, where van der waals interaction and electrostatic interaction are expected to be the main driving forces for the vesicle formation. However, so far the mechanism for various nonamphiphilic vesicles' formation is still unclear. Large amount of case-dependent knowledge is required before the mystery is completely solved.

Herein we report a case of nonamphiphilic vesicle which is driven predominantly by hydrogen bond in the dodecane/ $\beta$-cyclodextrin host-guest inclusion system. Cyclodextrins (CDs) are doughnut ring-like structures with a hydrophilic exterior and a hydrophobic cavity ${ }^{8}$. The hydrophobic cavity forms an ideal harbor in which poorly water-soluble molecules can shelter their most hydrophobic parts'. Therefore, CDs are able to form hostguest complexes with many molecules which change their self-assembling properties ${ }^{10-13}$. For instance, Jiang et al reported the formation of amphiphiles upon addition of CDs into a hydrophobic compound ${ }^{14}$. In most cases, the host-guest complexes based on CDs either self-assemble via hydrophobic interactions ${ }^{10,11}$ or lose the self-assembling abilities due to the increased solubility in water ${ }^{12,13}$.

However, our study revealed that the dodecane/ $\beta-C D$ inclusion system may self-assemble predominantly through hydrogen bonding into vesicles. Dodecane and $\beta$-CD was found to form $1: 2$ supramolecular complex dodecane@2 $\beta$-CD exclusively. Because the extending length of dodecane is much shorter than the sum of two $\beta$ $\mathrm{CDs}$, the dodecane molecule was completely buried in the channel formed by two $\beta$-CD molecules. Thus, dodecane was not directly involved in the interactions between the dodecane@2 $\beta$-CD supramolecular complexes, so that the self-assembly of dodecane@2 $\beta-C D$ is simply that of the channel type $\beta-C D$ dimers which is dominated by hydrogen bonds.

Hydrogen bonds occur very often in self-assembled one dimensional or network structures ${ }^{15-17}$. They also happen together with other weak interactions, such as hydrophobic effect and coordination, in various molecular self-assemblies ${ }^{18-20}$, but have never been reported being the major driving forces for the formation of vesicles. In this regard, the channel type $\beta$-CD dimer induced by dodecane (dodecane@ $2 \beta-C D$ ) has represented a new class of supramolecular building block that leads to nonamphiphilic vesicles. This is in clear contrast with the wellknown poor self-assembling behaviors of various CD monomers. It is expected that besides dodecane, other alkanes and compounds can also induce various channel type $\mathrm{CD}$ oligomers, such as dimer, trimer or tetramer, etc. These oligomers as powerful building blocks may further self-assemble into various advanced structures. We 
believe this result not only highlights a crucial feature of the supramolecular chemistry of CDs, but also sheds lights on the physics of self-assembling behavior. Meanwhile, it may open a new vista for material science.

\section{Results}

Exclusive formation of $1: 2$ inclusion complexes between dodecane and $\beta-C D$. Figure 1a shows the macroscopic phase behaviour of $\beta-C D$ solutions before and after addition of dodecane. The solution of $\beta-\mathrm{CD}$ is transparent at the concentration of $16 \mathrm{mM}$, whereas white precipitate was created upon the addition of $8 \mathrm{mM}$ dodecane, indicating the formation of inclusion complexes between $\beta-C D$ and dodecane ${ }^{21,22}$. The quantity of precipitates was almost doubled as the amount of dodecane was increased to $16 \mathrm{mM}$. However, mass analysis with ${ }^{1} \mathrm{H} \mathrm{NMR}^{23-25}$ revealed that the ratios between $\beta-\mathrm{CD}$ and dodecane in both precipitates were nearly $2: 1$ (see Supplementary Fig. 1). This means that the inclusion complex was predominantly dodecane@2 $\beta$ $\mathrm{CD}$ which is independent of the initial material ratio. This is in clear contrast with the inclusion complex formed between $\beta-C D$ and surfactant, where the resulting complex is mixing ratio dependent ${ }^{18,26}$. On the other hand, ESI-MS measurement showed the presence of supramolecular species [dodecane@2 $\left.\beta-\mathrm{CD}+\mathrm{CH}_{3} \mathrm{OH}+3 \mathrm{H}\right]^{3+}(\mathrm{m} / \mathrm{z}$ 824.89503 , the theoretical value $\mathrm{m} / \mathrm{z}$ 824.99867) (Fig. 1b), which further confirmed the formation of dodecane@2 $\beta$-CD. One may notice that the difference between the theoretical calculation and experimental result is much larger than the usual errors with a magnitude of several ppm in MS measurements. This larger error in our case is attributed to the following two reasons: 1) the large molecular mass for the [dodecane@2 $\left.\beta-\mathrm{CD}+\mathrm{CH}_{3} \mathrm{OH}+3 \mathrm{H}\right]^{3+}$ supramolecular species exceeds the upper limit of 2000 for the HT-ESIMS; 2) the charge neutral nature of the original dodecane@2 $\beta-C D$ supramolecular structures makes it difficult to obtain signals of charged molecular species so that the relative intensity has no direct relation with the content of the representing species ${ }^{27}$. As to the presence of $\mathrm{CH}_{3} \mathrm{OH}$, it is probable aroused from the hydrogen bonding between $\beta-\mathrm{CD}$ and $\mathrm{CH}_{3} \mathrm{OH}$, since the measurements were made in the methanol atmosphere.

The structure of dodecane@2 $\beta-C D$ verified with Small Angle Xray Scattering (SAXS). The formation of the $2: 1$ inclusion complex suggested that two $\beta$-CDs have been threaded with one dedecane chain to form channel type dimers, as demonstrated in Fig. 2a. This is further confirmed by the SAXS measurements. It is well-known that two possible crystalline structures, namely, cage-type and channel-type ${ }^{28}$, can be formed through $\beta$-CD/guest inclusion complexes. The cage-type structures display characteristic peaks at $6.8,9.1,9.4$, and $12.8 \mathrm{~nm}^{-1}$, while the channel-type ones exhibit characteristic peaks at 8.2 and $12.4 \mathrm{~nm}^{-1}$ in their XRD patterns ${ }^{18}$. In Fig. 2b, we show the SAXS pattern of the lyophilized white precipitates, which is dramatically different from that of cage-type $\beta$-CD powders, but are in perfect agreement with that of channel type ones.

The self-assembled structures based on the supramolecular dodecane@2及-CD complex. The regular scattering patterns indicate the formation of well-organized microstructures. SEM observation of the precipitates (Fig. 3a) revealed the formation of 'brick'-like structures. Large area of observation with light microscopy (Fig. 3b) also confirms the formation of polydispersed bricks from the basic supramolecular building block of dedecane@ $2 \beta$-CD. Moreover, it is interesting to find that vesicles were formed at low concentrations where precipitation was avoided. Figure $3 \mathrm{c}$ depicts the TEM image of the freeze-fractured sample (FF-TEM) for the $1 \mathrm{mM}$ sample which is diluted from the precipitated one in Fig. 1a. Vesicles with diameters of $50 \sim 200 \mathrm{~nm}$ were observed. Angle-dependent DLS measurements also revealed the presence of spherical structures with average radius of $100 \mathrm{~nm}$ (see Fig. 3d). The hollow nature of these spherical particles was verified with AFM observations (Fig. $4 \mathrm{a}-\mathrm{c}$ ). It is known that the extending length for dodecane and the height of $\beta-C D$ is about 1.2 and $0.8 \mathrm{~nm}$, respectively. This means that the dodecane should be completely buried in the hydrophobic channels formed by two $\beta$-CDs. Therefore, the thickness of the single layer of vesicle membrane under AFM should be around $1.6 \mathrm{~nm}$. For a dried unilamellar vesicle, the height of $3.2 \mathrm{~nm}$ is expected. This was indeed the case. First of all, the size for the spherical particles in Fig. 4a was consistent with that obtained in Fig. 3c and 3d. Most importantly, the sectional height profile in Fig. $4 \mathrm{~b}$ for two spheres were found being $\sim 3.2 \mathrm{~nm}$ and $1.6 \mathrm{~nm}$, respectively. The $3.2 \mathrm{~nm}$ thickness is obviously resulted from a collapsed unilamellar vesicle, while the one of $1.6 \mathrm{~nm}$ can be attributed to the free channel type $\beta$-CD dimers which didn't participate into self-assemble or be aroused from part of the broken membrane. Figure $4 \mathrm{c}$ shows there were also sectional height about $6.4 \mathrm{~nm}$ and $4.8 \mathrm{~nm}$, which corresponds to a collapsed and a broken double lamellar vesicle, respectively.

\section{Discussion}

The self-assembling behavior of dodecane@2 $\beta$-CD supramolecular inclusion complex is fairly amazing since most self-assembling

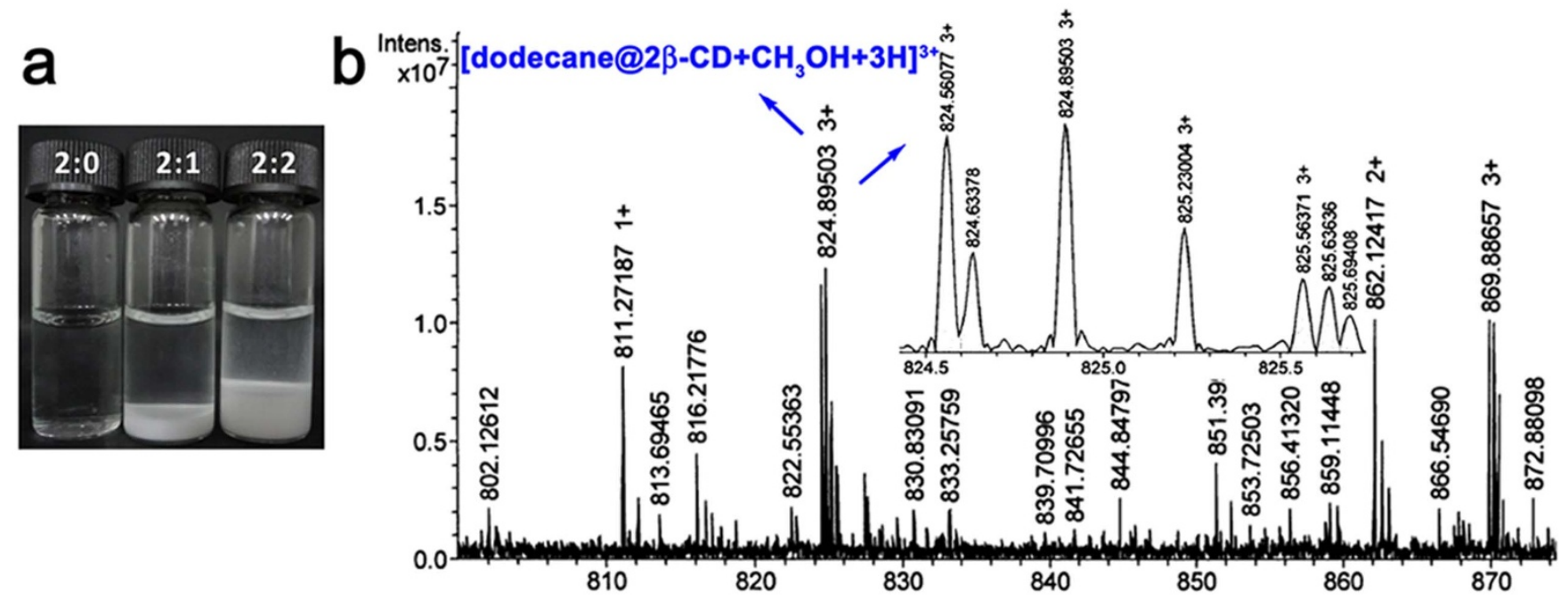

Figure $1 \mid$ (a) The macrographs of $\beta-C D\left(C_{\beta-C D}=16 \mathrm{mM}\right)$, dodecane/ $\beta$-CD mixed sample with the host-gust stoichiometries $2: 1$ and $2: 2$, respectively. (b) ESI-MS results of dodecane/ $\beta$-CD sample in positive mode. 

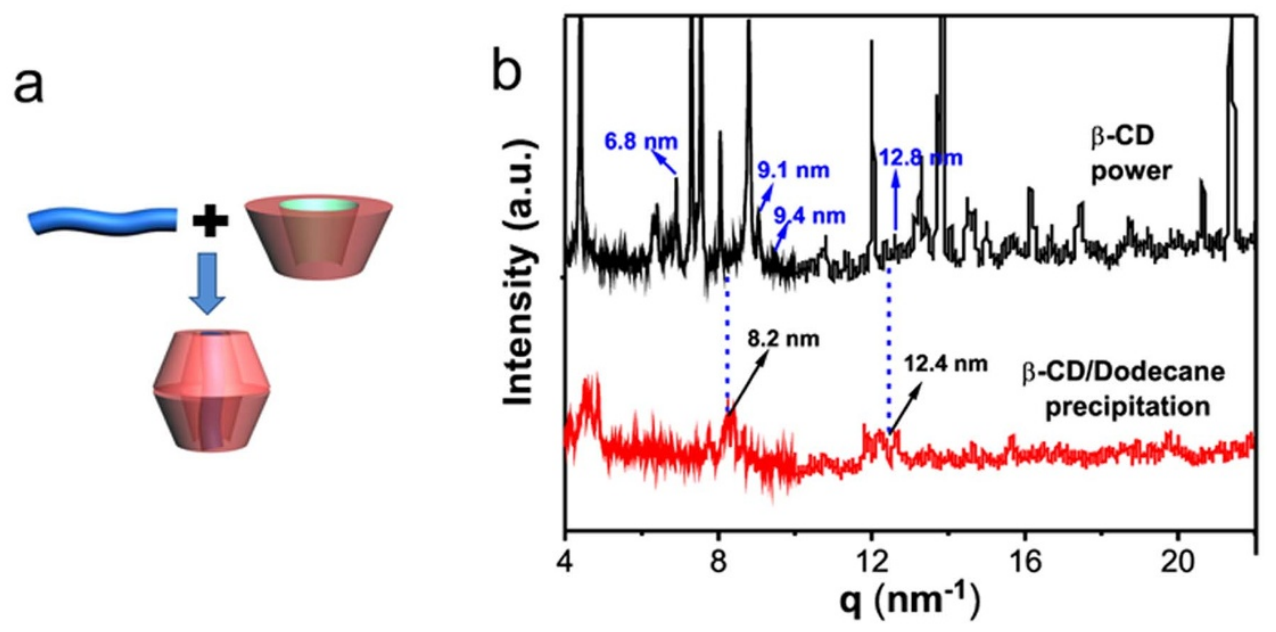

Figure $2 \mid$ (a) Illustration of the formation of channel type dimmers of $\beta$-CD induced by dodecane. (b) SAXS measurements for the lyophilized dodecane $/ \beta$-CD precipitates. $q=4 \pi \sin \theta / \lambda$.

systems require contradictory properties inside one building block. Typical examples are the amphiphilic surfactant and polymers. However, no hydrophobic portion can be found for the supramolecular building block of dodecane@2 $\beta$-CD since the $1.2 \mathrm{~nm}$ dodecane is completely buried in the $1.6 \mathrm{~nm}$ channel formed by two $\beta$-CDs. This means that the self-assembly of the dodecane@2 $\beta$-CD is a matter of self-assembly of the channel type dimer of $\beta$-CD. Then the driving force for the self-assembly formation is almost the same as that for the crystalline behavior of $\beta$-CD unimer. Namely, hydrogen bonding is crucial for the self-assembly of the dodecane@2 $\beta$-CD supramolecular building blocks ${ }^{29-31}$. To verify this point, urea, which is famous for its role in breaking hydrogen bonds, was added to the
$16 \mathrm{mM}$ system where precipitates exist. It was observed that the white precipitates disappeared immediately in the presence of $5 \mathrm{M}$ urea, (see Supplementary Figure S2), indicative of the disassembly of the bricks and verifying the importance of hydrogen bonds in keeping the self-assembles structures.

In regard to the formation of vesicles at low but bricks at high concentrations, it is possible that the different content of water in the self-assembled structures has resulted in different curvatures. At low concentrations, more water molecules are expected to bridge the dodecane@2 $\beta$-CD supramolecular building blocks. The uneven distribution of water molecules in the outer and inner side of the membrane leads to the curvature for vesicles. In contrast, at high enough
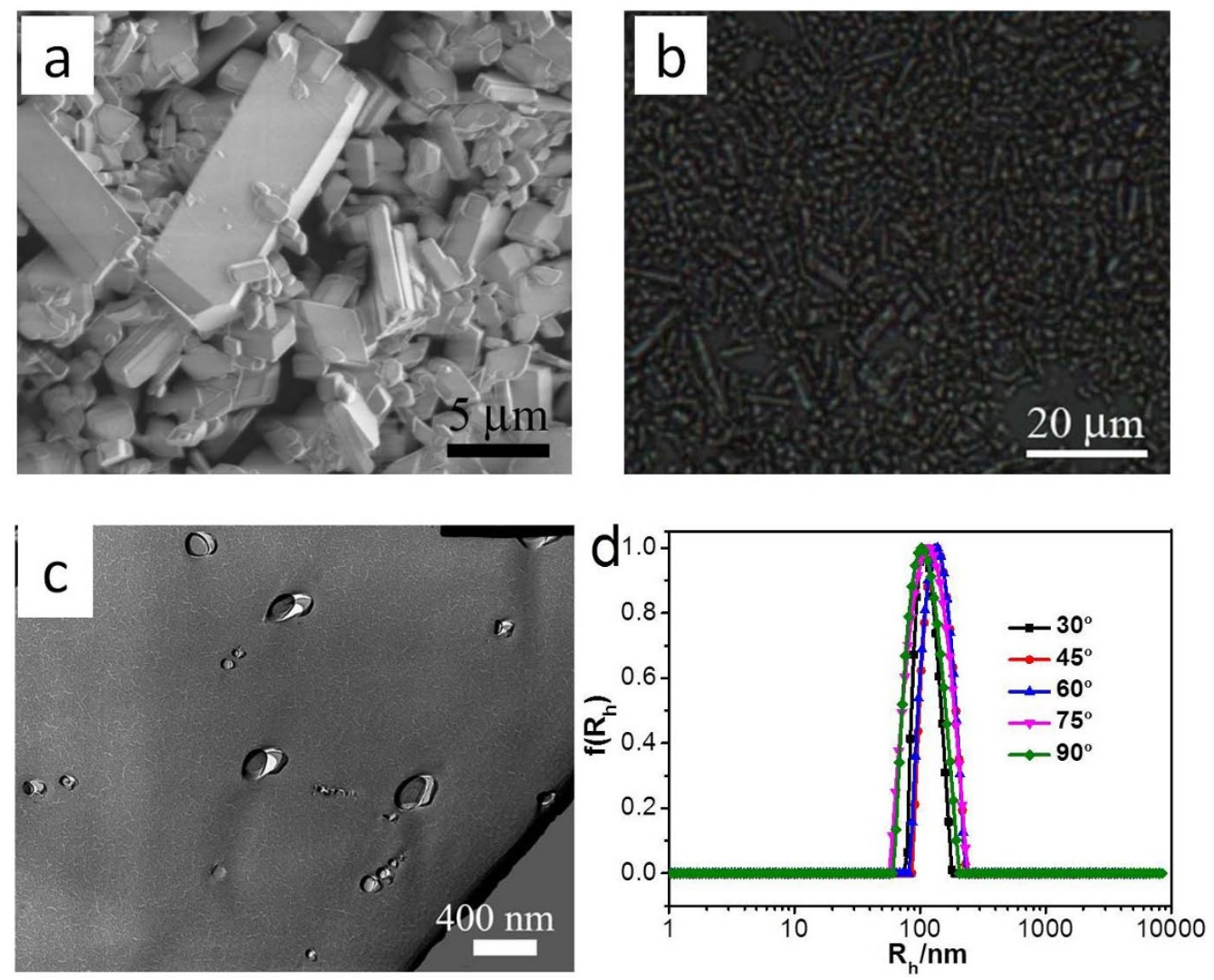

Figure $3 \mid$ (a) SEM and (b) Optical microscope images of the dodecane/ $\beta$-CD precipitates in $16 \mathrm{mM} \beta-\mathrm{CD} / 8 \mathrm{mM}$ dodecane system. (c) FF-TEM micrographs and d) DLS results for the vesicles formed in the $2 \mathrm{mM} \beta-\mathrm{CD} / 1 \mathrm{mM}$ dodecane system at various scattering angles. 

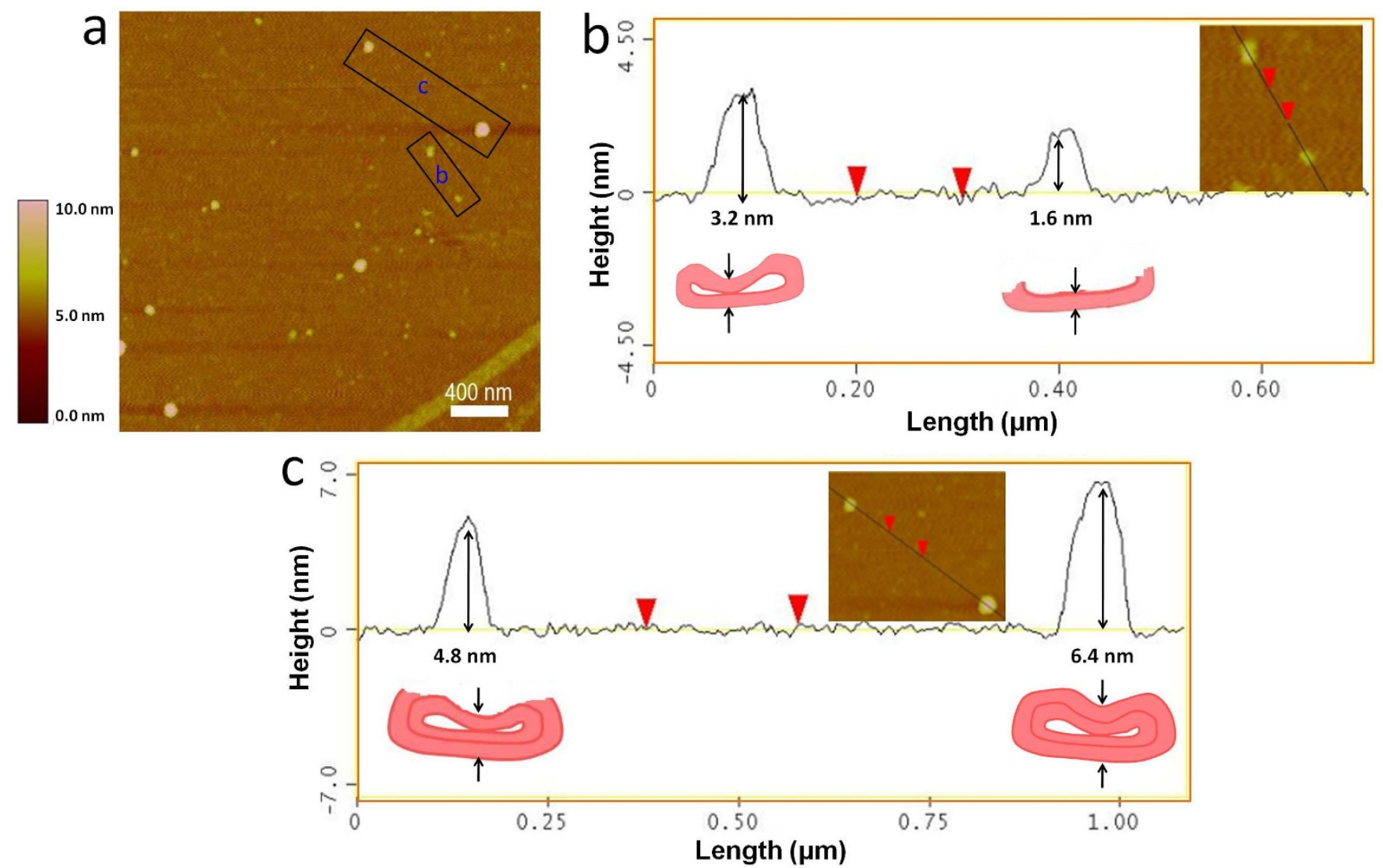

Figure $4 \mid$ (a) AFM images for vesicles formed in the dodecane@2 $\beta$-CD system $\left(\mathrm{C}_{\text {dodecane }}=1 \mathrm{mM}, \mathrm{C}_{\beta-\mathrm{CD}}=2 \mathrm{mM}\right)$. (b) Sectional height profile of a collapsed unilamellar vesicle and a broken unilamellar vesicle for region b in Figure 4a. (c) Sectional height profile of a collapsed bilamellar vesicle and a broken bilamellar vesicle for region c in Figure 4a.

concentrations, there are enough $\beta$-CD dimers in the system so that direct hydrogen bonding between the hydroxyl groups of neighboring $\beta$-CD is available. The parallel packing of the $\beta$-CD dimers allows formation of planar self-assemblies, which is brick in this case. As shown in Fig. 5, the wave number for the hydroxyl group in the 'bricks' shifts to a much lower value compared with that in the vesicles, which demonstrates that the hydrogen bond in the bricks is stronger than that in vesicles ${ }^{32}$. This means that the content of the hydrogen bonds between $\mathrm{H}_{2} \mathrm{O}$ and the $-\mathrm{OH}$ of $\beta-\mathrm{CD}$ in the vesicular

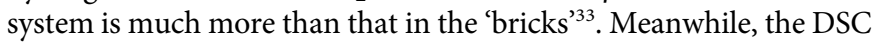
and TGA measurements also demonstrated that the structure water

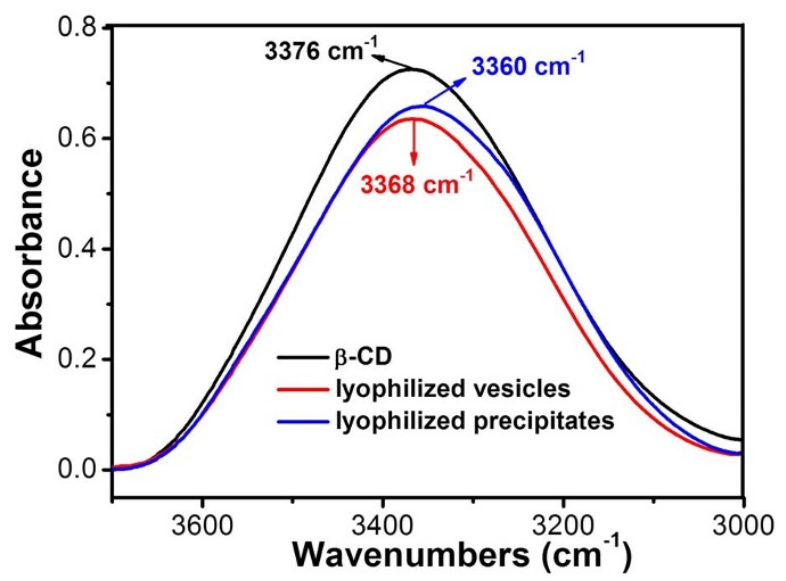

Figure $5 \mid$ The FT-IR spectra of $\beta$-CD, the lyophilized vesicles and lyophilized precipitates. have been lowered in the bricks (Fig. 6). The DSC curve for the lyophilized vesicles exhibits an endothermic peak centered at $45^{\circ} \mathrm{C}$. Upon slowing down the scanning rate from $2.0 \mathrm{~K} / \mathrm{min}$ to $0.5 \mathrm{~K} / \mathrm{min}$, DSC did not resolve the endothermic peak into separate events, indicating that all the water molecules are simultaneously eliminated. On the other hand, TGA analysis for the lyophilized vesicles shows that the weight losses of water are $13.2 \%$ (see Fig. 6a). This weight loss is attributed to the structural water of the vesicles ${ }^{34}$. That's to say, averagely each dodecane@ $2 \beta-C D$ supramolecular building block binds about 21 water molecules in order to form vesicles [(13.2/ $18.0) /(86.8 / 2440.3)=20.6]$. In contrast, the DSC curve for the lyophilized bricks has two endothermic peaks at $50^{\circ} \mathrm{C}$ and $80^{\circ} \mathrm{C}$ (Fig. 6b), indicating the presence of water molecules in different environments. TGA results show that the weight losses corresponding to these two peaks are $6.5 \%$ and $1.5 \%$, respectively. That's to say, the structural water in the bricks is only $8 \%$, which is much less than the content of water in vesicles, which is in good agreement with the deduction based on the FT-IR spectra in Fig. 5. It should be also noticed in Fig. 5 that both wave numbers for the $-\mathrm{OH}$ groups in the vesicles and the bricks are lower than that for the powder of $\beta$ $\mathrm{CD}$, indicating more water molecules have involved in the selfassembly of the $\beta$-CD dimers. It is possible the participation of water slows down the crystalline process so that well-defined structures are formed. In this point of view, the channel-type $\beta$-CD dimers can be viewed as a new class of building block which is different from the $\beta$ $\mathrm{CD}$ monomers. The whole process of self-assembly formation in the dodecane- $\beta$-CD system is illustrated in Fig. 7.

In conclusion, vesicles have been constructed with the channel type dimers of $\beta-\mathrm{CD}$ which is facilitated by dodecane. The addition of dodecane into the solution of $\beta$-CD allows the formation of dodecane@2 $\beta$-CD supramolecular building blocks where the 

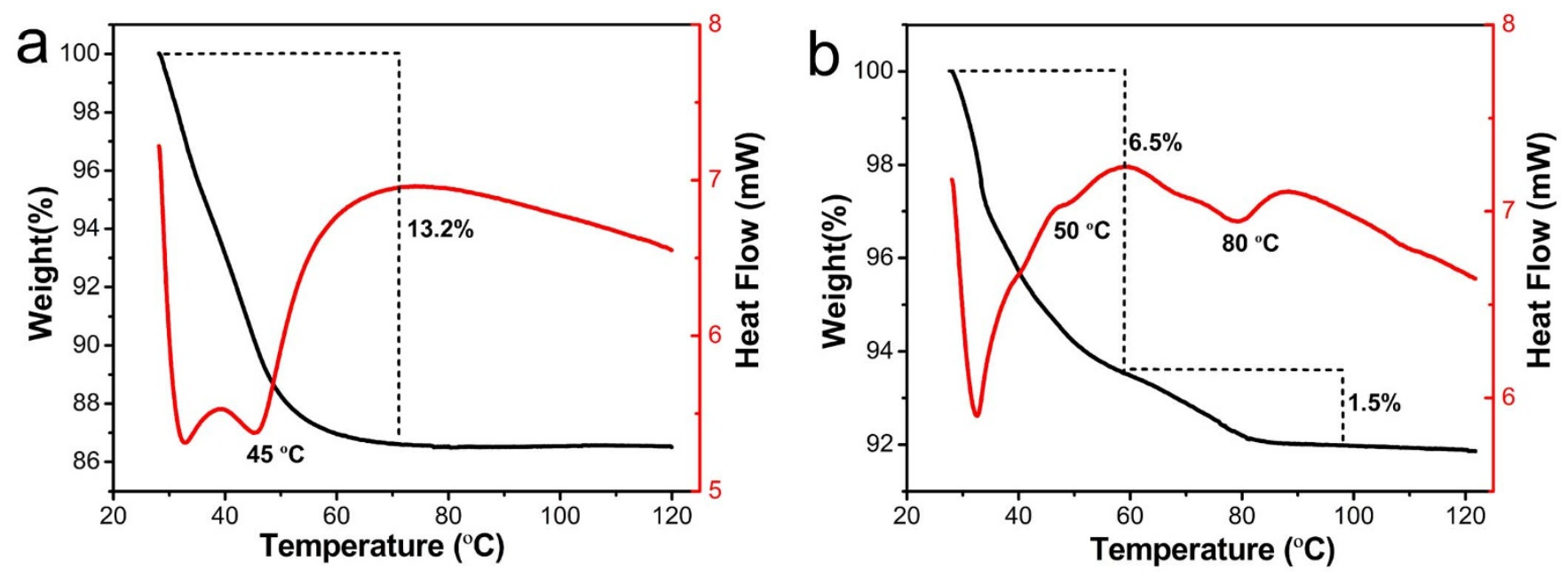

Figure 6 | The TGA and DSC results for the lyophilized vesicles (a) and precipitates (b), respectively.

dodecane was encaged into the cavity of the channel formed by two $\beta$-CDs. The channel type $\beta$-CD dimers then self-assemble mainly via hydrogen bonding. At low concentrations, more water molecules were involved in the hydrogen bonding and the uneven distribution of the water molecules in the outer and inner side of the membrane provided the curvature for vesicle formation. At high concentrations, the amount of water participated in the hydrogen bonding is much less so that no curvature can be formed thus plannar structures are formed. We expect that besides dodecane, other alkanes and compounds can also induce various channel type CD oligomers, such as dimer, trimer or tetramer, etc. These oligomers as powerful building blocks may further self-assemble into various advanced structures.

\section{Methods}

Materials. $\mathrm{n}$-Dodecane (99\%) were purchased from Alfa Aesar. $\beta$-Cyclodextrin ( $\beta$ $\mathrm{CD}$ ) with a water content of $14 \%$ were purchased from Sinopharm Chemical Reagent Co. Ultrapure water was used throughout the work.

Sample preparation. For the precipitate samples, dodecane was added into the $\beta$-CD solutions of $16 \mathrm{mM}$ at the initial host-guest stoichiometries of $2: 1$ and $2: 2$,

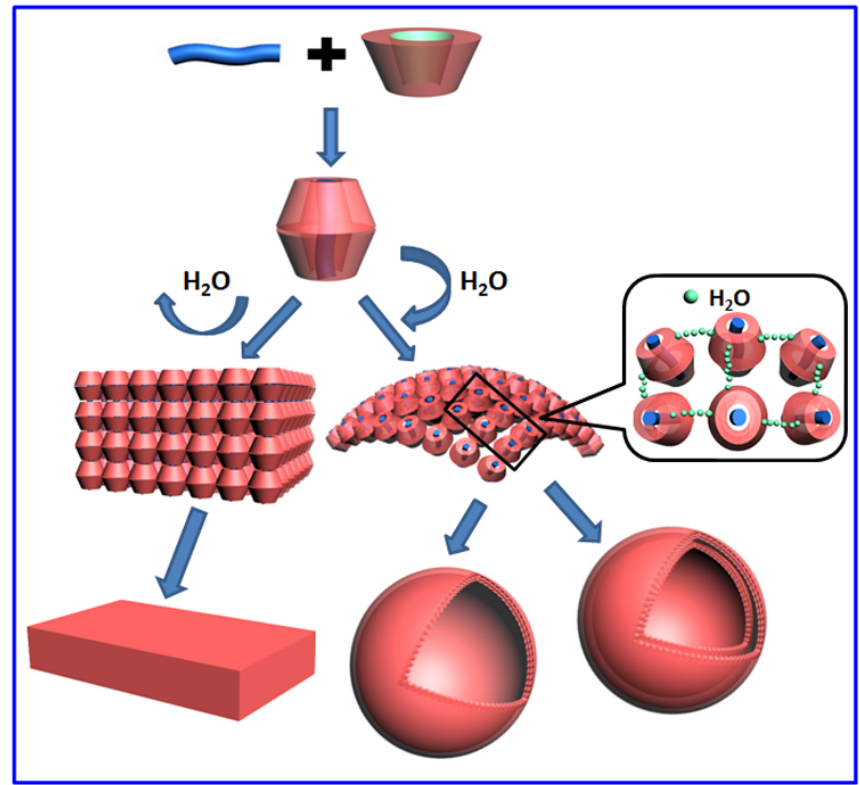

Figure $7 \mid$ Schematic illustration for the self-assembling behavior of the dimers of $2 \beta-C D$ threaded by one dodecane. The dimer is a supramolecular building block of dodecane@2 $\beta$-CD. respectively, and then the samples were vortex mixed sufficiently to create the white precipitate. After removing the supernatant solution, the dodecane $/ \beta$-CD precipitates from the initial host-guest stoichiometries of $2: 1$ and $2: 2$ were lyophilized to obtain white powders. For the vesicle sample, desired amounts of lyophilized dodecane/ $\beta$ CD precipitates and water were weighed into test tubes to give the dodecane@2 $\beta$-CD concentration of $1 \mathrm{mM}$. The resultant solutions were thermostatically incubated at $25^{\circ} \mathrm{C}$ (for at least $24 \mathrm{~h}$ ) to allow self-assembly formation.

${ }^{1}$ H NMR measurement. The lyophilized precipitates or vesicles were dissolved into DMSO for ${ }^{1} \mathrm{H}$ NMR measurements to determine the actual ratio of dodecane to $\beta$ $\mathrm{CD}$. The measurements were performed on an AVANCE III $500 \mathrm{M} \mathrm{Hz}$ NMR (Bruker, Switzerland). All the proton signals were calibrated with the TMS at $0.00 \mathrm{ppm}$. By comparing the integration between $\beta-\mathrm{CD}(\mathrm{H} 1$ protons, $\delta=4.83 \mathrm{ppm})$ and dodecane $\left(\mathrm{CH}_{3}\right.$ protons, $\left.\delta=0.83 \mathrm{ppm}\right)$, dodecance $/ \beta-\mathrm{CD}$ complex ratio can be determined.

Optical microscope observation. The dodecane/ $\beta$-CD precipitates were dropped on the slide and observed with an optical microscope (Olympus BX51TRF with DP72SET digital camera) equipped with polarizers in reflection mode under white-light illumination.

SEM observation. The dodecane $/ \beta-C D$ precipitates was mounted onto silicon wafer and air-dried before applied to SEM observation (SEM, Hitachi S4800, $5 \mathrm{kV}$ ).

SAXS measurements. The SAXS measurement of the $\beta$-CD power and lyophilized dodecane/ $\beta$-CD precipitates was conducted on a high-flux small-angle X-ray scattering instrument (SAXSess, Anton Paar) equipped with a Kratky blockcollimation system. A Philips PW3830 sealed-tube X-ray generator $(\mathrm{Cu} \mathrm{K} \alpha)$ was employed to simultaneously measure the small-angle and wide-angle X-ray scattering (WAXS and SAXS) of the samples. The scanning patterns extend to the high-angle range (the q range covered by the imaging plate is from 0.0 to $27.2 \mathrm{~nm}^{-1}, \mathrm{q}=4 \pi \sin \theta$ / $\lambda$, where $\lambda$ is the wavelength of $0.1542 \mathrm{~nm}$ and $2 \theta$ is the scattering angle). The scattering angles were calibrated with aluminum.

ESI-MS measurements. FT-MS measurements were carried out on an APEX IV FTMS (Bruker, USA). The operating conditions of the ESI source: positive ion mode; spray voltage $-3300 \mathrm{~V}$; capillary voltage $-3800 \mathrm{~V}$, capillary temperature $230^{\circ} \mathrm{C}$; skimmer1 $33.0 \mathrm{~V}$, skimmer2 $28.0 \mathrm{~V}$; sheath gas nitrogen pressure $0.3 \mathrm{bar}$. The lyophilized precipitates were dissolved in pure methanol with the concentration of $0.1 \mathrm{mM}$ and introduced via direct infusion at a flow rate of $3.00 \mu \mathrm{L} / \mathrm{min}$.

Dynamic light scattering (DLS). Dynamic light scattering (DLS) measurements were conducted on an ALV/DLS/SLS5022F light scattering-apparatus, equipped with a $22 \mathrm{~mW}$ He-Ne laser (632.8 $\mathrm{nm}$ wavelength) with a refractive index matching bath of filtered toluene surrounding the cylindrical scattering cell. The samples were filtered by $450 \mathrm{~nm}$ filters. The scattering angle was set from $30^{\circ}$ to $90^{\circ}$.

Freeze-fracture transmission electron microscopy (FF-TEM). A small amount of sample was placed on a $0.1 \mathrm{~mm}$ thick copper disk and then covered with a second copper disk. The sample was frozen by plunging into liquid propane cooled by liquid nitrogen. Fracturing and replication were performed on a freeze-fracture apparatus (BalzersBAF400, Germany) at $-140^{\circ} \mathrm{C}$. Pt/C was deposited at an angle of $45^{\circ}$ to shadow the replicas, and $\mathrm{C}$ was deposited at an angle of $90^{\circ}$ to consolidate the replicas. The resulting replicas were observed in a JEM-100CX II electron microscope (JEOL, Japan). 
Atomic force microscopy (AFM). AFM measurements in tapping mode under ambient conditions were conducted on a D3100 AFM (VEECO, USA). One drop of the dodecane/ $\beta$-CD solution was spin-coated on a mica surface, and then placed at room temperature to dry before AFM observation.

Fourier Transform Infrared (FT-IR). FT-IR measurements were performed on Nicolet Magna IR 750 equipped with an infrared microspectrography (Thermo Scientific Co., USA). The samples were frozen in liquid nitrogen and subsequently lyophilized for $48 \mathrm{~h}$ before FT-IR measurements.

TGA and DSC measurements. TGA and DSC measurements were conducted synchronously on a Q600SDT (Thermal Analysis Company, USA). The vesicle sample and brick sample were frozen in a liquid nitrogen bath followed by a vacuum freeze drying step before TGA and DSC measurements. The lyophilized samples were placed into the alumina crucible with air as the reference. The heating-rate was $2 \mathrm{~K} /$ min from 25 to $120^{\circ} \mathrm{C}$

1. Whitesides, G. M. \& Grzybowski, B. Self-assembly at all scales. Science 295, 2418-2421 (2002)

2. Coleman, A. C. et al. Light-induced disassembly of self-assembled vesicle-capped nanotubes observed in real time. Nat. nanotechnol. 6, 547-552 (2011).

3. Whitesides, G. M., Mathias, J. P. \& Seto, C. T. Molecular self-assembly and nanochemistry: a chemical strategy for the synthesis of nanostructures. Science 254, 1312-1319 (1991)

4. Southall, N. T., Dill, K. A. \& Haymet, A. A view of the hydrophobic effect. J. Phys. Chem. B 106, 521-533 (2002).

5. Liu, T., Diemann, E., Li, H., Dress, A. W. \& Müller, A. Self-assembly in aqueous solution of wheel-shaped Mo154 oxide clusters into vesicles. Nature 426, 59-62 (2003).

6. Li, D., Zhang, J., Landskron, K. \& Liu, T. Spontaneous self-assembly of metalorganic cationic nanocages to form monodisperse hollow vesicles in dilute solutions. J. Am. Chem. Soc. 130, 4226-4227 (2008).

7. Wu, L., Lal, J., Simon, K. A., Burton, E. A. \& Luk, Y.-Y. Nonamphiphilic assembly in water: polymorphic nature, thread structure, and thermodynamic incompatibility. J. Am. Chem. Soc. 131, 7430-7443 (2009).

8. Szejtli, J. Introduction and general overview of cyclodextrin chemistry. Chem. Rev. 98, 1743-1754 (1998).

9. Junquera, E., Tardajos, G. \& Aicart, E. Effect of the presence of. beta.-cyclodextrin on the micellization process of sodium dodecyl sulfate or sodium perfluorooctanoate in water. Langmuir 9, 1213-1219 (1993).

10. Bojinova, T. et al. Complexes between $\beta$-cyclodextrin and aliphatic guests as new noncovalent amphiphiles: formation and physicochemical studies. Langmuir 19, 5233-5239 (2003).

11. Jing, B. et al. Self-Assembly Vesicles Made from a Cyclodextrin Supramolecular Complex. Chem-Eur J 13, 9137-9142 (2007).

12. Saenger, W. \& Müller-Fahrnow, A. Cyclodextrins increase surface tension and critical micelle concentrations of detergent solutions. Angew. Chem. Int. Ed. Engl. 27, 393-394 (1988).

13. Jiang, L., Yan, Y., Drechsler, M. \& Huang, J. Enzyme-triggered model selfassembly in surfactant-cyclodextrin systems. Chem. Commun. 48, 7347-7349 (2012).

14. Zou, J., Tao, F. \& Jiang, M. Optical switching of self-assembly and disassembly of noncovalently connected amphiphiles. Langmuir 23, 12791-12794 (2007).

15. Bakota, E. L., Sensoy, O., Ozgur, B., Sayar, M. \& Hartgerink, J. D. Self-assembling multidomain peptide fibers with aromatic cores. Biomacromolecules 14, 1370-1378 (2013)

16. Jiang, L. et al. “Annular Ring” microtubes formed by SDS@ 2 $\beta-C D$ complexes in aqueous solution. Soft Matter 6, 1731-1736 (2010).

17. Fichman, G. \& Gazit, E. Self-assembly of short peptides to form hydrogels: Design of building blocks, physical properties and technological applications. Acta biomaterialia 10, 1671-1682 (2014).

18. Jiang, L., Peng, Y., Yan, Y. \& Huang, J. Aqueous self-assembly of SDS@ 2 $\beta$-CD complexes: lamellae and vesicles. Soft Matter 7, 1726-1731 (2011).

19. Rest, C. et al. Self-Assembly and (Hydro) gelation Triggered by Cooperative $\pi-\pi$ and Unconventional $\mathrm{CH} \cdots \mathrm{X}$ Hydrogen Bonding Interactions. Angew. Chem. Int. Ed. 53, 700-705 (2014)

20. Shen, Z., Wang, T. \& Liu, M. H-bond and $\pi-\pi$ stacking directed self-assembly of two-component supramolecular nanotubes: tuning length, diameter and wall thickness. Chem. Commun. 50, 2096-2099 (2014).
21. Sanemasa, I. et al. Preparation of Cyclodextrin Precipitates with Volatile Hydrocarbons in Aqueous Media, and Determination of Hydrocarbon/ Cyclodextrin Molar Rations in the Precipitates. Bull. Chem. Soc. Jpn. 66, 1424-1429 (1993).

22. Dou, Z. P., Xing, H. \& Xiao, J. X. Hydrogenated and Fluorinated Host-Guest Surfactants: Complexes of Cyclodextrins with Alkanes and Fluoroalkyl-Grafted Alkanes. Chem-Eur J 17, 5373-5380 (2011).

23. Wilson, L. D. \& Verrall, R. E. A 1H NMR study of cyclodextrin-hydrocarbon surfactant inclusion complexes in aqueous solutions. Can. J. Chem. 76, 25-34 (1998).

24. Freitag, M., Gundlach, L., Piotrowiak, P. \& Galoppini, E. Fluorescence Enhancement of Di-p-tolyl Viologen by Complexation in Cucurbit [7] uril. J. Am Chem. Soc. 134, 3358-3366 (2012).

25. Tao, W. et al. A linear-hyperbranched supramolecular amphiphile and its selfassembly into vesicles with great ductility. J. Am. Chem. Soc. 134, 762-764 (2011)

26. Jiang, L., Yan, Y. \& Huang, J. Zwitterionic surfactant/cyclodextrin hydrogel: microtubes and multiple responses. Soft Matter 7, 10417-10423 (2011).

27. Guo, M. et al. Studies on the non-covalent complexes between oleanolic acid and cyclodextrins using electrospray ionization tandem mass spectrometry. J. Mass Spectrom. 38, 723-731 (2003).

28. Harada, A., Okada, M., Li, J. \& Kamachi, M. Preparation and characterization of inclusion complexes of poly (propylene glycol) with cyclodextrins. Macromolecules 28, 8406-8411 (1995).

29. Saenger, W. \& Steiner, T. Cyclodextrin inclusion complexes: host-guest interactions and hydrogen-bonding networks. Acta Crystallogr. Sect. A: Found. Crystallogr. 54, 798-805 (1998).

30. Zhou, C. et al. Self-Assembly of Nonionic Surfactant Tween 20@ 2 $\beta$-CD Inclusion Complexes in Dilute Solution. Langmuir 29, 13175-13182 (2013).

31. Bas, G. L. \& Tsoucaris, G. Two-dimensional hydrogen bond network in $\beta$ cyclodextrin complexes. Supramol. Chem. 4, 13-16 (1994).

32. Ke, D., Zhan, C., Li, A. D. \& Yao, J. Morphological Transformation between Nanofibers and Vesicles in a Controllable Bipyridine-Tripeptide Self-Assembly. Angew. Chem. 123, 3799-3803 (2011).

33. Manas, E. S., Getahun, Z., Wright, W. W., DeGrado, W. F. \& Vanderkooi, J. M. Infrared spectra of amide groups in $\alpha$-helical proteins: Evidence for hydrogen bonding between helices and water. J. Am. Chem. Soc. 122, 9883-9890 (2000).

34. Cheruzel, L. E. et al. Structures and Solid-State Dynamics of One-Dimensional Water Chains Stabilized by Imidazole Channels. Angew. Chem. 115, 5610-5613 (2003).

\section{Acknowledgments}

This work was supported by the Natural Science Foundation of China (21173011, 21273013), the Foundation for Innovative Research Group of the Natural Science Foundation of China (51121091) and the National Basic Research Program of China (973 Program, 2013CB933800).

\section{Author contributions}

C.C.Z. performed all the experiments and involved in writing; X.H.C. and Q.Z. provided useful help in ESI-MS and FF-TEM experiments; Y.Y. proposed the study and revised the manuscript; J.B.H. and J.D.W. participated useful discussions. All authors reviewed the manuscript

\section{Additional information}

Supplementary information accompanies this paper at http://www.nature.com/ scientificreports

Competing financial interests: The authors declare no competing financial interests.

How to cite this article: Zhou, C. et al. Self-Assembly of Channel Type $\beta$-CD Dimers Induced by Dodecane. Sci. Rep. 4, 7533; DOI:10.1038/srep07533 (2014).

This work is licensed under a Creative Commons Attribution-NonCommercialNoDerivs 4.0 International License. The images or other third party material in this article are included in the article's Creative Commons license, unless indicated otherwise in the credit line; if the material is not included under the Creative Commons license, users will need to obtain permission from the license holder in order to reproduce the material. To view a copy of this license, visit http:// creativecommons.org/licenses/by-nc-nd/4.0/ 